PROCEEDINGS OF THE

AMERICAN MATHEMATICAL SOCIETY

Volume 134, Number 2, Pages 581-590

S 0002-9939(05)08219-5

Article electronically published on August 12, 2005

\title{
EQUIVALENCE OF TOPOLOGIES AND BOREL FIELDS FOR COUNTABLY-HILBERT SPACES
}

\author{
JEREMY J. BECNEL
}

(Communicated by Jonathan M. Borwein)

\begin{abstract}
We examine the main topologies-weak, strong, and inductiveplaced on the dual of a countably-normed space and the $\sigma$-fields generated by these topologies. In particular, we prove that for certain countably-Hilbert spaces the strong and inductive topologies coincide and the $\sigma$-fields generated by the weak, strong, and inductive topologies are equivalent.
\end{abstract}

\section{INTRODUCTION AND OBJECTIVES}

In this paper we study the weak, strong, and inductive topologies on the dual of a countably-normed space. We see that under certain conditions the strong and inductive topologies coincide (and are also equivalent with the Mackey topology, which is introduced later). We also examine and compare the $\sigma$-fields generated by these topologies to see that under reasonable conditions all the $\sigma$-fields are in fact equivalent.

Although these results have been used implicitly or explicitly (see for instance [5]) in the literature, there appears to be no efficient or complete proof published or readily accessible without having to work through a vast literature. It is the purpose of this paper to present proofs of these results in a largely self-contained manner. For this reason we have included here proofs of various well-known results; these proofs also serve the function of setting up arguments and notation for our principal objectives.

\section{TOPOLOGICAL VECTOR SPACES}

In this section we review the basic notions of topological vector spaces and provide proofs of a few useful results.

2.1. Topological preliminaries. Let $E$ be a real vector space. A vector topology $\tau$ on $E$ is a topology such that addition $E \times E \rightarrow E:(x, y) \mapsto x+y$ and scalar multiplication $\mathbb{R} \times E \rightarrow E:(t, x) \mapsto t x$ are continuous. If $E$ is a complex vector space we require that $\mathbb{C} \times E \rightarrow E:(\alpha, x) \mapsto \alpha x$ be continuous. A topological vector space is a vector space equipped with a vector topology.

A local base of a vector topology $\tau$ is a family of open sets $\left\{U_{\alpha}\right\}_{\alpha \in I}$ containing 0 such that if $W$ is any open set containing 0 , then $W$ contains some $U_{\alpha}$. A set $W$ that contains an open set containing $x$ is called a neighborhood of $x$. If $U$ is any

Received by the editors September 2, 2004.

2000 Mathematics Subject Classification. Primary 57N17; Secondary 60H40.

(C)2005 American Mathematical Society Reverts to public domain 28 years from publication 
open set and $x$ any point in $U$, then $U-x$ is an open neighborhood of 0 and hence contains some $U_{\alpha}$, and so $U$ itself contains a neighborhood $x+U_{\alpha}$ of $x$. Doing this for each point $x$ of $U$, we see that each open set is the union of translates of the local base sets $U_{\alpha}$.

If $\mathcal{U}_{x}$ denotes the set of all neighborhoods of a point $x$ in a topological space $X$, then $\mathcal{U}_{x}$ has the following properties:

1. $x \in U$ for all $U \in \mathcal{U}_{x}$,

2. if $U \in \mathcal{U}_{x}$ and $V \in \mathcal{U}_{x}$, then $U \cap V \in \mathcal{U}_{x}$,

3. if $U \in \mathcal{U}_{x}$ and $U \subset V$, then $V \in \mathcal{U}_{x}$,

4. if $U \in \mathcal{U}_{x}$, then there is some $V \in \mathcal{U}_{x}$ with $U \in \mathcal{U}_{y}$ for all $y \in V$. (Taking $V$ to be the interior of $U$ is sufficient.)

Conversely, if $X$ is any set and a non-empty collection of subsets $\mathcal{U}_{x}$ is given for each $x \in X$, then when the conditions above are satisfied by the $\mathcal{U}_{x}$, exactly one topology can be defined on $X$ in such a way to make $\mathcal{U}_{x}$ the set of neighborhoods of $x$ for each $x \in X$. This topology consists of all $V \subset X$ such that for each $x \in V$ there is a $U \in \mathcal{U}_{x}$ with $U \subset V[6$.

We are primarily concerned with locally convex spaces; a locally convex space is a topological vector space which has a local base consisting of convex sets.

In a topological vector space there is the notion of bounded sets. A set $D$ in a topological vector space is said to be bounded, if for every neighborhood $U$ of 0 there is some $\lambda>0$ such that $D \subset \lambda U$. If $\left\{U_{\alpha}\right\}_{\alpha \in I}$ is a local base, then it is easily seen that $D$ is bounded if and only if to each $U_{\alpha}$ there corresponds $\lambda_{\alpha}>0$ with $D \subset \lambda_{\alpha} U_{\alpha}[6]$.

A set $A$ in a vector space $E$ is said to be absorbing if given any $x \in E$ there is an $\eta$ such that $x \in \lambda A$ for all $|\lambda| \geq \eta$. The set $A$ is balanced if, for all $x \in A, \lambda x \in A$ whenever $|\lambda| \leq 1$. Also, a set $A$ in a vector space $E$ is symmetric if $-A=A$.

2.2. Bases in topological vector spaces. Here we prove some general, but very useful, results about local bases for topological vector spaces [6].

Lemma 2.1. Every topological vector space E has a base of balanced neighborhoods.

Proof. Let $U$ be a neighborhood of 0 in $E$. Consider the function $h: \mathbb{C} \times E \rightarrow E$ given by $h(\lambda, x)=\lambda x$. Since $E$ is a topological vector space, $h$ is continuous at $\lambda=0, x=0$. So there is a neighborhood $V$ and $\epsilon>0$ with $\lambda x \in U$ for $|\lambda| \leq \epsilon$ and $x \in V$. Hence $\lambda V \subset U$ for $|\lambda| \leq \epsilon$. Therefore $\frac{e}{\alpha} V \subset U$ for all $\alpha$ with $|\alpha| \geq 1$. Thus $\epsilon V \subset U^{\prime}=\bigcap_{|\alpha| \geq 1} \alpha U \subset U$. Now since $V$ is a neighborhood of 0 , so is $\epsilon V$. Hence $U^{\prime}$ is a neighborhood of 0 . If $x \in U^{\prime}$ and $0<|\lambda| \leq 1$, then for $|\alpha| \geq 1$, we have $x \in \frac{\alpha}{\lambda} U$. So $\lambda x \in \alpha U$ for $|\lambda| \leq 1$. Hence $\lambda x \in U^{\prime}$. Therefore $U^{\prime}$ is balanced.

Lemma 2.2. Let $E$ be a vector space. Let $\mathcal{B}$ be a collection of subsets of $E$ satisfying:

I. if $U, V \in \mathcal{B}$, then there exist $W \in \mathcal{B}$ with $W \subset U \cap V$,

II. if $U \in \mathcal{B}$ and $\lambda \neq 0$, then $\lambda U \in \mathcal{B}$,

III. if $U \in \mathcal{B}$, then $U$ is balanced, convex, and absorbing.

Then there is a topology making $E$ a locally convex topological vector space with $\mathcal{B}$ the base of neighborhoods of 0.

Proof. Let $\mathcal{A}$ be the set of all subsets of $E$ that contain a set of $\mathcal{B}$. For each $x$ take $x+\mathcal{A}$ to be the set of neighborhoods of $x$. We need to see that properties $1-4$ are satisfied from subsection 2.1 
For property 1, we have to show $x \in A$ for all $A \in x+\mathcal{A}$. Note that since each $U$ is absorbing, there exists a non-zero $\lambda$ such that $0 \in \lambda U$. But then $0 \in \lambda^{-1} \lambda U=U$. So each $U \in \mathcal{B}$ contains 0 . Hence $x \in A$ for all $A \in x+\mathcal{A}$.

For property 2, we have to show that if $A, B \in \mathcal{A}$, then $(x+A) \cap(x+B) \in x+\mathcal{A}$ for each $x \in E$. Recall $U \subset A$ and $V \subset B$ for some $U, V \in \mathcal{B}$. So $U \cap V \subset A \cap B$. By the first hypothesis, there is a $W \in \mathcal{B}$ with $W \subset U \cap V \subset A \cap B$. Thus $A \cap B \in \mathcal{A}$ and hence $(x+A) \cap(x+B) \in x+\mathcal{A}$ for each $x \in E$.

Next, property 3 is clear from the definition of $\mathcal{A}$, since if $A \in \mathcal{A}$ and $A \subset B$, then $B \in \mathcal{A}$.

Finally for property 4 , we must show that if $x+A \in x+\mathcal{A}$, there is a $V \in x+\mathcal{A}$ with $x+A \in y+\mathcal{A}$ for all $y \in V$. If $A \in \mathcal{A}$ take a $U \in \mathcal{B}$ with $U \subset A$. Now we show that $x+A$ is a neighborhood of each point $y \in x+\frac{1}{2} U$. Since $y \in x+\frac{1}{2} U$ we have $y-x \in \frac{1}{2} U$. Thus $y-x+\frac{1}{2} U \subset \frac{1}{2} U+\frac{1}{2} U \subset A$. Hence $y+\frac{1}{2} U \subset x+A$. Thus $x-y+A \supset \frac{1}{2} U$, so $x-y+A \in \mathcal{A}$. Therefore $y+x-y+A=x+A \in y+\mathcal{A}$.

To prove continuity of addition, let $U \in \mathcal{B}$. Then if $x \in a+\frac{1}{2} U$ and $y \in b+\frac{1}{2} U$, we have $x+y \in a+b+U$. To see that scalar multiplication, $\lambda x$, is continuous at $x=a, \lambda=\alpha$, we should find $\delta_{1}$ and $\delta_{2}$ such that $\lambda x-\alpha a \in U$ whenever $|\lambda-\alpha|<\delta_{1}$ and $x \in a+\delta_{2} U$. Since $U$ is absorbing, there is a non-zero $\eta$ with $a \in \eta U$. Take $\delta_{1}$ so that $0<\delta_{1}<\frac{1}{2 \eta}$ and take $\delta_{2}$ so that $0<\delta_{2}<\frac{1}{2\left(|\alpha|+\delta_{1}\right)}$. Using the fact that $U$ is balanced

$$
\begin{aligned}
\lambda x-\alpha a=\lambda(x-a)+(\lambda-\alpha) a & \in\left(|\alpha|+\delta_{1}\right) \delta_{2} U+\delta_{1} \eta U \\
& \subset \frac{1}{2} U+\frac{1}{2} U \subset U .
\end{aligned}
$$

Thus we are done.

2.3. Topologies generated by families of topologies. Let $\left\{\tau_{\alpha}\right\}_{\alpha \in I}$ be a collection of vector topologies on a vector space $E$. It is natural and useful to consider the least upper bound topology $\tau$, i.e. the coarsest topology containing all sets of $\bigcup_{\alpha \in I} \tau_{\alpha}$. We present the following useful fact:

Fact 2.3. The least upper bound topology $\tau$ of a collection $\left\{\tau_{\alpha}\right\}_{\alpha \in I}$ of vector topologies is again a vector topology. If $\left\{W_{\alpha, i}\right\}_{i \in I_{\alpha}}$ is a local base for $\tau_{\alpha}$, then a local base for $\tau$ is obtained by taking all finite intersections of the form $W_{\alpha_{1}, i_{1}} \cap \cdots \cap W_{\alpha_{n}, i_{n}}$. (See [8, Example 9.2 (b).)

\section{Countably-normed spaces}

We begin with the basic definition of a countably-normed space and a countablyHilbert space.

Definition 3.1. Let $V$ be a topological vector space over $\mathbb{C}$ with topology given by a family of norms $\left\{|\cdot|_{n} ; n=1,2, \ldots\right\}$. Then $V$ is a countably-normed space. The space $V$ is called a countably-Hilbert space if each $|\cdot|_{n}$ is an inner product norm and $V$ is complete with respect to its topology.

Remark 3.2. By considering the new norms $\|v\|_{n}=\left(\sum_{k=1}^{n}|v|_{k}^{2}\right)^{\frac{1}{2}}$ we may and will assume that the family of norms $\left\{|\cdot|_{n} ; n=1,2, \ldots\right\}$ is increasing, i.e.

$$
|v|_{1} \leq|v|_{2} \leq \cdots \leq|v|_{n} \leq \cdots, \forall v \in V .
$$


If $V$ is a countably-normed space, we denote the completion of $V$ in the norm $|\cdot|_{n}$ by $V_{n}$. Then $V_{n}$ is by definition a Banach space. Also in light of Remark 3.2 we can assume that

$$
V \subset \cdots \subset V_{n+1} \subset V_{n} \subset \cdots \subset V_{1}
$$

Lemma 3.3. The inclusion map from $V_{n+1}$ into $V_{n}$ is continuous.

Proof. Take an open neighborhood of 0 in $V_{n}$ given by $B_{n}(0, \epsilon)=\left\{v \in V_{n} ;|v|_{n}<\epsilon\right\}$. Let $i_{n+1, n}: V_{n+1} \rightarrow V_{n}$ be the inclusion map. Now

$$
i_{n+1, n}^{-1}\left(B_{n}(0, \epsilon)\right)=\left\{v \in V_{n+1} ;|v|_{n}<\epsilon\right\} \supset B_{n+1}(0, \epsilon) \text { since }|v|_{n} \leq|v|_{n+1} .
$$

Therefore $i_{n+1, n}$ is continuous.

Proposition 3.4. Let $V$ be a countably-normed space. Then $V$ is complete if and only if $V=\bigcap_{n=1}^{\infty} V_{n}$.

Proof. Suppose $V=\bigcap_{n=1}^{\infty} V_{n}$ and $\left\{v_{k}\right\}_{k=1}^{\infty}$ is Cauchy in $V$. By definition of the topology on $V,\left\{v_{k}\right\}_{k=1}^{\infty}$ is Cauchy in $V_{n}$ for all $n$. Since $V_{n}$ is complete, a limit $v^{(n)}$ exist in $V_{n}$. Since the inclusion map $i_{n+1, n}: V_{n+1} \rightarrow V_{n}$ is continuous and

$$
V \subset \cdots \subset V_{n+1} \subset V_{n} \subset \cdots \subset V_{1}
$$

all the $v^{(n)}$ are the same and belong to each $V_{n}$. Thus this common limit is in $V=$ $\bigcap_{n=1}^{\infty} V_{n}$. Let us call this element $v \in V$. Since for all $m, \lim _{k \rightarrow \infty}\left|v_{k}-v^{(n)}\right|_{m}=0$, then we have that $v=\lim _{k \rightarrow \infty} v_{k}$ in $V$. Thus $V$ is complete.

Conversely, let $V$ be complete and take $v \in \bigcap_{n=1}^{\infty} V_{n}$. Using that $V$ is dense in $V_{n}$, for each $n$ we can find $v_{n} \in V$ such that $\left|v-v_{n}\right|_{n}<\frac{1}{n}$. Now for $k<n$ we have $\left|v-v_{n}\right|_{k} \leq\left|v-v_{n}\right|_{n}<\frac{1}{n}$. Thus $\lim _{n \rightarrow \infty}\left|v-v_{n}\right|_{k}=0$. This gives us that $\left\{v_{n}\right\}$ is Cauchy with respect to $|\cdot|_{k}$ for all $k$, and hence Cauchy in the topology of $V$.

Let $\bar{v}=\lim _{n \rightarrow \infty} v_{n}$ in $V$. Since for all $k$ we have $\bar{v}, v \in V_{k}$ and $\lim _{n \rightarrow \infty}\left|\bar{v}-v_{n}\right|_{k}=$ 0 , we have that $v=\bar{v}$. Thus $v \in V$ and we have $V \supset \bigcap_{n=1}^{\infty} V_{n}$. That $V \subset \bigcap_{n=1}^{\infty} V_{n}$ is obvious, since $V \subset V_{n}$ for all $n$.

3.1. Open sets in $V$. In light of Fact 2.3. we see that a local base for $V$ is given by sets of the form

$$
B=B_{n_{1}}\left(\epsilon_{1}\right) \cap B_{n_{2}}\left(\epsilon_{2}\right) \cap \cdots \cap B_{n_{k}}\left(\epsilon_{k}\right),
$$

where $B_{n_{i}}\left(\epsilon_{i}\right)=\left\{v \in V ;|v|_{n_{i}}<\epsilon_{i}\right\}$ is the $|\cdot|_{n_{i}}$ unit ball of radius $\epsilon_{i}$ in $V$.

Proposition 3.5. Let $V$ be a countably-normed space. For every element $B$ of the local base for $V$ there exist $n$ and $\epsilon>0$ such that $B_{n}(\epsilon) \subset B$.

Proof. Let $B=B_{n_{1}}\left(\epsilon_{1}\right) \cap B_{n_{2}}\left(\epsilon_{2}\right) \cap \cdots \cap B_{n_{k}}\left(\epsilon_{k}\right)$ be an element of the local base for $V$. Take $n=\max _{1 \leq j \leq k} n_{j}$ and $\epsilon=\min _{1 \leq j \leq k} \epsilon_{j}$. Observe that $B_{n}(\epsilon) \subset B$ since for $v \in B_{n}(\epsilon)$ we have $|v|_{n_{j}} \leq|v|_{n}<\epsilon \leq \epsilon_{j}$ for any $j \in\{1,2, \ldots, k\}$ and so $v \in B$.

3.2. The dual. Let $V$ be a countably-normed space associated with an increasing sequence of norms $\left\{|\cdot|_{n}\right\}_{n=1}^{\infty}$. Let $V_{n}$ be the completion of $V$ with respect to the norm $|\cdot|_{n}$. We denote the dual space of $V$ by $V^{\prime}$. Let $\langle\cdot, \cdot\rangle$ denote the canonical bilinear pairing of $V^{\prime}$ and $V$.

Proposition 3.6. The dual of a countably-normed space $V$ is given by $V^{\prime}=$ $\bigcup_{n=1}^{\infty} V_{n}^{\prime}$ and we have the inclusions

$$
V_{1}^{\prime} \subset \cdots \subset V_{n}^{\prime} \subset V_{n+1}^{\prime} \subset \cdots \subset V^{\prime} .
$$


Proof. (つ) Take $v^{\prime} \in V_{n}^{\prime}$. Then $v^{\prime}$ is continuous on $V_{n}$ with topology coming from the norm $|\cdot|_{n}$. Thus $v^{\prime}$ is continuous on $V$, since $V \subset V_{n}$ and the norm $|\cdot|_{n}$ is one of the norms generating the topology on $V$.

(C) Take $v^{\prime} \in V^{\prime}$. The set $N_{v^{\prime}}(1)=\left\{v \in V ;\left|\left\langle v^{\prime}, v\right\rangle\right|<1\right\}$ is open in $V$ since $v^{\prime}$ is continuous on $V$. By Proposition 3.5 we have $B_{n}(\epsilon) \subset N_{v^{\prime}}(1)$ for some $n$ and some $\epsilon>0$. Thus for all $v \in V$ with $|v|_{n}<\epsilon$ we have that $\left|\left\langle v^{\prime}, v\right\rangle\right|<1$. Since $V$ is dense in $V_{n}$, if $v \in V_{n}$ and $|v|_{n} \leq \epsilon$, then $\left|\left\langle v^{\prime}, v\right\rangle\right| \leq 1$. Hence $v^{\prime} \in V_{n}^{\prime}$.

The fact that $V_{n}^{\prime} \subset V_{n+1}^{\prime}$ follows from $V_{n+1} \subset V_{n}$ and the fact that the inclusion maps $i_{n+1, n}: V_{n+1} \rightarrow V_{n}$ are continuous (see Lemma 3.3).

\section{TOPOlOGiEs ON THE DUAL SPACE}

We now briefly introduce four of the most common topologies placed on the dual of a countably-normed space. We examine the weak, strong, inductive, and Mackey topologies.

4.1. Weak topology. The weak topology is the simplest topology placed on the dual of a countably-normed space. It is defined as follows.

Definition 4.1. The weak topology on the dual $V^{\prime}$ of a topological vector space $V$ is the coarsest vector topology on $V^{\prime}$ such that the functional $\langle\cdot, v\rangle$ is continuous for any $v \in V$.

Remark 4.2. Just as we have defined the weak topology on $V^{\prime}$, we can define an analogous topology on $V$. This topology has as its local base sets of the form

$$
N\left(v_{1}^{\prime}, v_{2}^{\prime}, \ldots, v_{k}^{\prime} ; \epsilon\right)=\left\{v \in V ;\left|\left\langle v_{j}^{\prime}, v\right\rangle\right|<\epsilon, 1 \leq j \leq k\right\} .
$$

This topology is referred to as the weak topology on $V$.

As with any vector topology, we are interested in a useful local base.

Proposition 4.3. The weak topology on $V^{\prime}$ has a local base of neighborhoods given by sets of the form

$$
N\left(v_{1}, v_{2}, \ldots, v_{k} ; \epsilon\right)=\left\{v^{\prime} \in V^{\prime} ;\left|\left\langle v^{\prime}, v_{j}\right\rangle\right|<\epsilon, 1 \leq j \leq k\right\} .
$$

Proof. In order for $\langle\cdot, v\rangle$ to be continuous for all $v \in V$ we need $\langle\cdot, v\rangle$ to be continuous at 0 . Or equivalently, we require that $N(v ; \epsilon)$ be open for each $\epsilon>0$. Hence for each $v \in V$ we form the topology $\tau_{v}$ on $V$ given by the local base $\{N(v ; \epsilon)\}_{\epsilon>0}$. The weak topology is the least upper bound topology for the family $\left\{\tau_{v}\right\}_{v \in V}$ (see subsection 2.31). Thus a local base for the weak topology is given by sets of the form

$$
N\left(v_{1}, v_{2}, \ldots, v_{k} ; \epsilon\right)=N\left(v_{1} ; \epsilon\right) \cap N\left(v_{2} ; \epsilon\right) \cap \cdots \cap N\left(v_{k} ; \epsilon\right),
$$

where $v_{1}, v_{2}, \ldots, v_{k} \in V$.

Now suppose $V$ is a countably-normed space with notation as in Section 3 ,

Proposition 4.4. The inclusion map $i_{n}^{\prime}: V_{n}^{\prime} \rightarrow V^{\prime}$ is continuous when $V^{\prime}$ is given the weak topology and $V_{n}^{\prime}$ has the dual-norm topology.

Proof. Consider the weak base neighborhood $N(v ; \epsilon)$, where $v \in V$. Observe that $i_{n}^{\prime-1}(N(v ; \epsilon))=\left\{v^{\prime} \in V_{n}^{\prime} ;\left|\left\langle v^{\prime}, v\right\rangle\right|<\epsilon\right\}$. Since $v \in V \subset V_{n}$ we have that the functional $\langle\cdot, v\rangle$ is continuous on $V_{n}^{\prime}$. (Since $V_{n}$ is a Banach space, $V_{n} \subset V_{n}^{\prime \prime}$.) Thus $\left\{v^{\prime} \in V_{n}^{\prime} ;\left|\left\langle v^{\prime}, v\right\rangle\right|<\epsilon\right\}$ is open in $V_{n}^{\prime}$. 
4.2. Strong topology. Using the notion of bounded sets in a countably-normed space $V$ we can define the strong topology on $V^{\prime}$.

Definition 4.5. The strong topology on the dual $V^{\prime}$ of a topological vector space $V$ is defined to be the topology with a local base given by sets of the form

$$
N(D ; \epsilon)=\left\{v^{\prime} \in V^{\prime} ; \sup _{v \in D}\left|\left\langle v^{\prime}, v\right\rangle\right|<\epsilon\right\},
$$

where $D$ is any bounded subset of $V$ and $\epsilon>0$.

Taking $D$ to be a finite set such as $\left\{v_{1}, v_{2}, \ldots, v_{k}\right\}$, it is clear that the strong topology is finer than the weak topology.

Proposition 4.6. The inclusion map $i_{n}^{\prime}: V_{n}^{\prime} \rightarrow V^{\prime}$ is continuous when $V^{\prime}$ is given the strong topology and $V_{n}^{\prime}$ has the dual-norm topology.

Proof. Consider the neighborhood $N(D ; \epsilon)=\left\{v^{\prime} \in V^{\prime} ; \sup _{v \in D}\left|\left\langle v^{\prime}, v\right\rangle\right|<\epsilon\right\}$, where $D$ is bounded in $V$ and $\epsilon>0$. Now $i_{n}^{\prime-1}(N(D ; \epsilon))=\left\{v^{\prime} \in V_{n}^{\prime} ; \sup _{v \in D}\left|\left\langle v^{\prime}, v\right\rangle\right|<\epsilon\right\}$. Since $D$ is bounded we have $\sup _{v \in D}|v|_{n}=M<\infty$. Take $v_{0}^{\prime}$ in $i_{n}^{\prime-1}(N(D ; \epsilon))$ and let $c_{0}=\sup _{v \in D}\left|\left\langle v_{0}^{\prime}, v\right\rangle\right|<\epsilon$. Consider the open set $B\left(v_{0}^{\prime}, \frac{\epsilon-c_{0}}{M+1}\right)=\left\{v^{\prime} \in\right.$ $\left.V_{n}^{\prime} ;\left|v^{\prime}-v_{0}^{\prime}\right|_{-n}<\frac{\epsilon-c_{o}}{M+1}\right\}$. We assert that $B\left(v_{0}^{\prime}, \frac{\epsilon-c_{0}}{M+1}\right) \subset i_{n}^{\prime-1}(N(D ; \epsilon))$.

Take $v^{\prime} \in B\left(v_{0}^{\prime}, \frac{\epsilon-c_{0}}{M+1}\right)$. Then $\left|v^{\prime}-v_{0}^{\prime}\right|_{-n}<\frac{\epsilon-c_{0}}{M+1}$. This gives us the following:

$$
\sup _{v \in D, v \neq 0}\left|\left\langle v^{\prime}-v_{0}^{\prime}, \frac{v}{|v|_{n}}\right\rangle\right|<\frac{\epsilon-c_{0}}{M+1} .
$$

Thus $\sup _{v \in D}\left|\left\langle v^{\prime}-v_{0}^{\prime}, v\right\rangle\right|<\epsilon-c_{0}$, since $|v|_{n} \leq M$ when $v \in D$. From this we see that $\sup _{v \in D}\left|\left\langle v^{\prime}, v\right\rangle\right|<\epsilon$. Therefore $v^{\prime} \in i_{n}^{\prime-1}(N(D ; \epsilon))$.

4.3. Inductive limit topology. Suppose $\left\{\left(W_{n},|\cdot|_{n}\right) ; n \geq 1\right\}$ is a sequence of normed spaces with $W_{n}$ continuously included in $W_{n+1}$ for all $n$. Form the space $W=\bigcup_{n=1}^{\infty} W_{n}$ and endow $W$ with the finest locally convex vector topology such that for each $n$ the inclusion map $i_{n}: W_{n} \rightarrow W$ is continuous. The existence of such a topology is shown in Theorem 4.7. This topology is called the inductive limit topology on $W$, and $W$ is said to be the inductive limit of the sequence $\left\{\left(W_{n},|\cdot|_{n}\right) ; n \geq 1\right\}$.

4.4. Local base. It will be useful to work with a local base for the inductive limit topology.

Theorem 4.7. Suppose $W$ is the inductive limit of the sequence of normed spaces $\left\{\left(W_{n},|\cdot|_{n}\right) ; n \geq 1\right\}$. A local base for $W$ is given by the set $\mathcal{B}$ of all balanced convex subsets $U$ of $W$ such that $i_{n}^{-1}(U)$ is a neighborhood of 0 in $W_{n}$ for all $n$.

Proof. We first apply Lemma 2.2 to see the set $\mathcal{B}$ is in fact a local base for $W$. Take $U, V \in \mathcal{B}$, then clearly $U \cap V \in \mathcal{B}$. Now if $U \in \mathcal{B}$, then it is easy to see that $\alpha U \in \mathcal{B}$ for $\alpha \neq 0$. Finally we show $U \in \mathcal{B}$ is absorbing. Note that $i_{n}^{-1}(U)$ is absorbing in $W_{n}$ (since $W_{n}$ is a normed space and $i_{n}^{-1}(U)$ is open in $W_{n}$ ). Thus $U$ absorbs all points of $W_{n}=i_{n}\left(W_{n}\right) \subset W$. Since $W=\bigcup_{n=1}^{\infty} W_{n}, U$ absorbs $W$. Thus we see that $\mathcal{B}$ is a base of neighborhoods for a locally convex vector topology on $W$.

It is fairly straightforward to see that $\mathcal{B}$ gives us the finest locally convex vector topology making all the $i_{n}: W_{n} \rightarrow W$ continuous: Let $\tau$ be a locally convex vector topology on $W$ making all the $i_{n}$ continuous. Take a convex neighborhood (of 0 ) $U$ in $\tau$. By Lemma 2.1 we can assume $U$ is balanced. Since each $i_{n}$ is continuous, we have that $i_{n}^{-1}(U)$ is a neighborhood in $W_{n}$. Thus $U \in \mathcal{B}$. 
Corollary 4.8. Suppose $W$ is the inductive limit of the sequence of normed spaces $\left\{\left(W_{n},|\cdot|_{n}\right) ; n \geq 1\right\}$. A local base for $W$ is given by the balanced convex hulls of sets of the form $\bigcup_{n=1}^{\infty} i_{n}\left(B_{n}\left(\epsilon_{n}\right)\right.$ ) (where $\left.B_{n}\left(\epsilon_{n}\right)=\left\{x \in W_{n} ;|x|_{n}<\epsilon_{n}\right\}\right)$.

Proof. Let $U$ be the balanced convex hull of the set $\bigcup_{n=1}^{\infty} i_{n}\left(B_{n}\left(\epsilon_{n}\right)\right)$ in $W$. Then $B_{n}\left(\epsilon_{n}\right) \subset i_{n}^{-1}(U)$. So $i_{n}^{-1}(U)$ is a neighborhood of 0 in $W_{n}$. By Theorem $4.7 \mathrm{such}$ a $U$ is a neighborhood in $W$.

Now if $U$ is any balanced convex neighborhood of 0 in $W$, then $i_{n}^{-1}(U)$ contains a neighborhood $B_{n}\left(\epsilon_{n}\right)$. Hence $i_{n}\left(B_{n}\left(\epsilon_{n}\right)\right) \subset U$. Since $U$ is convex and balanced, the balanced convex hull of $\bigcup_{n=1}^{\infty} i_{n}\left(B_{n}\left(\epsilon_{n}\right)\right)$ is contained in $U$. Thus the sets described form a local base for $W$.

Let $V$ be a countably-normed space. Then $V^{\prime}$, the dual of $V$, can be regarded as the inductive limit of the sequence of normed spaces $\left\{\left(V_{n}^{\prime},|\cdot|{ }_{-n}\right) ; n \geq 1\right\}$. (Recall that we have $V^{\prime}=\bigcup_{n=1}^{\infty} V_{n}^{\prime}$.) In light of Proposition 4.6 and Proposition 4.4 we see that

the inductive limit topology on $V^{\prime}$ is finer than the strong and weak topology on $V^{\prime}$.

4.5. Mackey topology. In order to talk about the Mackey topology we need the following notion.

Definition 4.9. Let $\mathcal{D}$ be a non-empty set of bounded subsets of a topological vector space $E$ with dual $E^{\prime}$. The topology of uniform convergence on the sets of $\mathcal{D}$ is the topology with subbasis neighborhoods of 0 given by

$$
N(D ; \epsilon)=\left\{v^{\prime} \in E^{\prime} ; \sup _{v \in D}\left|\left\langle v^{\prime}, v\right\rangle\right|<\epsilon\right\},
$$

where $D \in \mathcal{D}$ and $\epsilon>0$. This is also referred to as the topology of $\mathcal{D}$-convergence on $E^{\prime}$.

From the definition we see that a local base neighborhood for the topology of $\mathcal{D}$-convergence on a vector space $E$ with dual $E^{\prime}$ looks like

$$
N\left(D_{1} ; \epsilon_{1}\right) \cap N\left(D_{2} ; \epsilon_{2}\right) \cap \cdots \cap N\left(D_{k} ; \epsilon_{k}\right),
$$

where $D_{j} \in \mathcal{D}$ and $\epsilon_{j}>0$ for all $1 \leq j \leq k$. We now state the following theorem without proof:

Theorem 4.10 (Mackey-Arens). Suppose that under a locally convex vector topology $\tau, E$ is a Hausdorff space. Then $E$ has dual $E^{\prime}$ under $\tau$ if and only if $\tau$ is a topology of uniform convergence on a set of balanced convex weakly-compact subsets of $E^{\prime}$.

For a proof of this result see Section 21.4 in [4. Using this theorem we can define the Mackey topology as follows:

Definition 4.11. Let $E$ be a topological vector space with dual $E^{\prime}$. The Mackey topology on $E$ is the topology of uniform convergence on all balanced convex weaklycompact subsets of $E^{\prime}$.

Remark 4.12. From this discussion we see that the Mackey topology on $E$ has a local base given by

$$
N(C ; \epsilon)=\left\{v \in E ; \sup _{v^{\prime} \in C}\left|\left\langle v^{\prime}, v\right\rangle\right|<\epsilon\right\},
$$

where $\epsilon>0$ and $C$ is a balanced convex weakly-compact set in $E^{\prime}$. 
4.6. Comparing the topologies on $V^{\prime}$. Let us make the following notational convention throughout this section:

Notation. Let $V$ be a countably-normed space with dual $V^{\prime}$. The weak topology, strong topology, inductive limit topology, and Mackey topology on $V^{\prime}$ will be denoted by $\tau_{w}, \tau_{s}, \tau_{i}$, and $\tau_{m}$, respectively.

Proposition 4.13. Let $V$ be a countably-normed space with dual $V^{\prime}$. Then the strong topology $\tau_{s}$ is finer than the Mackey topology $\tau_{m}$ on $V^{\prime}$.

Proof. The topology $\tau_{s}$ is by definition the topology of uniform convergence on all bounded sets in $V$. Note that every weakly bounded set in $V$ is bounded, and every weakly-compact set in $V$ is weakly bounded. Thus $\tau_{m} \subset \tau_{s}$.

Lemma 4.14. Let $V$ be a countably-normed space with dual $V^{\prime}$. Then $V^{\prime}$ is Hausdorff in the weak topology $t_{w}$, and hence in the strong, Mackey, and inductive limit topologies.

Proof. Take $v^{\prime} \in V^{\prime}$. We must find a neighborhood of 0 in $\tau_{w}$ that does not contain $v^{\prime}$. Take $v \in V$ such that $\left|\left\langle v^{\prime}, v\right\rangle\right| \neq 0$. Let $\left|\left\langle v^{\prime}, v\right\rangle\right|=\lambda \neq 0$. Consider the set $N\left(v ; \frac{\lambda}{2}\right)=\left\{v^{\prime} \in V^{\prime} ;\left|\left\langle v^{\prime}, v\right\rangle\right|<\frac{\lambda}{2}\right\}$. This set cannot contain $v^{\prime}$.

Lemma 4.15. Let $V$ be a countably-Hilbert space with dual $V^{\prime}$. Then the dual of $V^{\prime}$ is $V$ when $V^{\prime}$ is given the weak, strong, Mackey, or inductive limit topology.

Proof. Consider $v \in V$ and the corresponding linear functional $\hat{v}$ on $V^{\prime}$ given by $\left\langle\hat{v}, v^{\prime}\right\rangle=\left\langle v^{\prime}, v\right\rangle$, where $v^{\prime} \in V^{\prime}$. Observe that $\langle\hat{v}, \cdot\rangle$ is continuous since $\left\{v^{\prime} \in\right.$ $\left.V^{\prime} ;\left|\left\langle v^{\prime}, v\right\rangle\right|<\epsilon\right\}$ is open in the weak topology (and hence the strong, Mackey, and inductive limit topologies). Also note that if $\hat{u}=\hat{v}$, then $\left\langle v^{\prime}, v\right\rangle=\left\langle v^{\prime}, u\right\rangle$ for all $v^{\prime} \in V^{\prime}$. So $v=u$ and by the Hahn-Banach Theorem the correspondence $v \rightarrow \hat{v}$ is injective.

We now show that the correspondence $v \rightarrow \hat{v}$ is surjective as a map of $V$ into the dual of $V^{\prime}$ with respect to any of the four topologies. Take $v^{\prime \prime} \in V^{\prime \prime}$, the dual of $V^{\prime}$. Then $v^{\prime \prime}$ is continuous on $V^{\prime}$. Since $V^{\prime}=\bigcup_{n=1}^{\infty} V_{n}^{\prime}$, we have that $v^{\prime \prime} \in V_{n}^{\prime \prime}$ for all $n$. (Here we have used that $i_{n}^{\prime}: V_{n}^{\prime} \rightarrow V^{\prime}$ is continuous in all four topologies by Proposition 4.4, Proposition 4.6, Proposition 4.13, and the definition of the inductive limit topology.) But $V_{n}=V_{n}^{\prime \prime}$ since $V_{n}$ is a Hilbert space. Thus $v^{\prime \prime}$ can be considered as an element of $V_{n}$ for all $n$. Since $V$ is complete, we have that $\bigcap_{n=1}^{\infty} V_{n}=V$ by Proposition 3.4. Thus $v^{\prime \prime} \in V$ and we have that $v \rightarrow \hat{v}$ is surjective.

Theorem 4.16. Let $V$ be a countably-Hilbert space with dual $V^{\prime}$. Then the inductive, strong, and Mackey topologies on $V^{\prime}$ are equivalent (i.e. $\left.\tau_{s}=\tau_{i}=\tau_{m}\right)$.

Proof. By Lemma 4.14 and Lemma 4.15 we have that $V^{\prime}$ is Hausdorff and has dual $V$ under the topologies $\tau_{s}, \tau_{i}$, and $\tau_{m}$. Thus we can apply Theorem 4.10 to $V^{\prime}$. (In the theorem we are taking $V^{\prime}$ as $E$ and $V$ as $E^{\prime}$.) This gives us that $\tau_{i}$ and $\tau_{s}$ are topologies of uniform convergence on a set of balanced convex weakly-compact subsets of $V$. However, the Mackey topology, $\tau_{m}$, is the finest such topology. Thus $\tau_{s} \subset \tau_{m}$ and $\tau_{i} \subset \tau_{m}$. However, by Proposition 4.13 we have $\tau_{m} \subset \tau_{s}$. Thus $\tau_{s}=\tau_{m}$. Likewise, we have $\tau_{i} \subset \tau_{m}=\tau_{s}$, and, by Proposition 4.6 and the definition of the inductive limit topology on $V^{\prime}$, we have $\tau_{s} \subset \tau_{i}$ (see (4.1)). Therefore $\tau_{s}=\tau_{m}=\tau_{i}$. 


\section{BOREL FIELD}

In this section our aim is to discuss the $\sigma$-fields on $V^{\prime}$ generated by the three topologies (strong, weak, and inductive). We will see that under certain conditions all the three $\sigma$-fields coincide. The standing assumption throughout this section is that $V$ is a countably-Hilbert space with a countable dense subset $Q_{o}$. From the definition of the topology on $V$ it follows that $Q_{o}$ is also dense as a subset of $V_{n}$. On each $V_{n}^{\prime} \subset V^{\prime}$ define the sets $F_{n}\left(\frac{1}{k}\right)$ for all $k$ as

$$
F_{n}\left(\frac{1}{k}\right)=\left\{v^{\prime} \in V_{n}^{\prime} ; \sup _{v \in Q}\left|\left\langle v^{\prime}, \frac{v}{|v|_{n}}\right\rangle\right|<\frac{1}{k}\right\},
$$

where $Q=Q_{o}-\{0\}$.

Recall that the local base for the dual-norm topology on $V_{n}^{\prime}$ is given by the sets

$$
N_{n}(\epsilon)=\left\{v^{\prime} \in V_{-n}^{\prime} ;\left|v^{\prime}\right|_{-n}<\epsilon\right\},
$$

where $\epsilon>0$. Observe that in $V_{n}^{\prime}$ we have $F_{n}\left(\frac{1}{k}\right)=N_{n}\left(\frac{1}{k}\right)$ for all $k$, because $Q$ is dense in $V_{n}$. Moreover, the collection $\left\{F_{n}\left(\frac{1}{k}\right)\right\}_{k=1}^{\infty}$ forms a local base in $V_{n}^{\prime}$.

Since each $V_{n}$ is a separable Hilbert space, so is its dual $V_{n}^{\prime}$. Let $Q_{n}^{\prime}$ be a countable dense subset in $V_{n}^{\prime}$.

Proposition 5.1. The collection $\left\{x^{\prime}+F_{n}\left(\frac{1}{k}\right) \mid x^{\prime} \in Q_{n}^{\prime}, 1 \leq k<\infty\right\}$ is a basis for the topology of $V_{n}^{\prime}$.

Proof. Consider an open set $U \subset V_{n}^{\prime}$ and an element $v^{\prime}$ in $U$. So there is a $k$ such that $v^{\prime}+F_{n}\left(\frac{1}{k}\right) \subset U$. Take $x^{\prime} \in Q_{n}^{\prime}$ such that $\left|x^{\prime}-v^{\prime}\right|_{-n}<\frac{1}{2 k}$.

Observe that $x^{\prime}+F_{n}\left(\frac{1}{2 k}\right) \subset v^{\prime}+F_{n}\left(\frac{1}{k}\right)$ : Take any $w^{\prime} \in F_{n}\left(\frac{1}{2 k}\right)$, and we have

$$
\begin{aligned}
\sup _{v \in Q}\left|\left\langle x^{\prime}-v^{\prime}+w^{\prime}, \frac{v}{|v|_{n}}\right\rangle\right| & \leq\left|x^{\prime}-v^{\prime}\right|_{-n}+\sup _{v \in Q}\left|\left\langle w^{\prime}, \frac{v}{|v|_{n}}\right\rangle\right| \\
& <\frac{1}{2 k}+\frac{1}{2 k}=\frac{1}{k} .
\end{aligned}
$$

This gives us that $x^{\prime}-v^{\prime}+F_{n}\left(\frac{1}{2 k}\right) \subset F_{n}\left(\frac{1}{k}\right)$ or equivalently $x^{\prime}+F_{n}\left(\frac{1}{2 k}\right) \subset v^{\prime}+F_{n}\left(\frac{1}{k}\right)$. Also $v^{\prime} \in x^{\prime}+F_{n}\left(\frac{1}{2 k}\right)$ since $\left|x^{\prime}-v^{\prime}\right|_{-n}<\frac{1}{2 k}$.

In summary we have that $v^{\prime} \in x^{\prime}+F_{n}\left(\frac{1}{2 k}\right) \subset v^{\prime}+F_{n}\left(\frac{1}{k}\right) \subset U$. Therefore the collection $\left\{x^{\prime}+F_{n}\left(\frac{1}{k}\right) \mid x^{\prime} \in Q_{n}^{\prime}, 1 \leq k<\infty\right\}$ is a basis for $V_{n}^{\prime}$

Lemma 5.2. Let $\sigma\left(\tau_{w}\right)$ be the Borel $\sigma$-field on $V^{\prime}$ induced by the weak topology. Then $F_{n}\left(\frac{1}{k}\right)$ is in $\sigma\left(\tau_{w}\right)$ for all positive integers $k$ and $n$.

Proof. Observe $F_{n}\left(\frac{1}{k}\right)=\left\{v^{\prime} \in V_{-n}^{\prime} ;\left|v^{\prime}\right|_{-n}<\frac{1}{k}\right\}=\left\{v^{\prime} \in V^{\prime} ;\left|v^{\prime}\right|_{-n}<\frac{1}{k}\right\}$. Note that $F_{n}\left(\frac{1}{k}\right)$ can be expressed as $F_{n}\left(\frac{1}{k}\right)=\bigcup_{r \in S} \bigcap_{v \in Q_{n}^{\prime}} N\left(\frac{v}{|v|_{n}} ; r\right)$, where $N\left(\frac{v}{|v|_{n}} ; r\right)=$ $\left\{v^{\prime} \in V^{\prime} ;\left|\left\langle v^{\prime}, v\right\rangle\right|<\frac{v}{|v|_{n}}\right\} r$ and $S=\left\{r \in \mathbb{Q} ; 0<r<\frac{1}{k}\right\}$. Therefore $F_{n}\left(\frac{1}{k}\right)$ is expressible using countable unions and intersections of the weakly open sets $N\left(\frac{v}{|v|_{n}} ; r\right)$. Hence $F_{n}\left(\frac{1}{k}\right)$ is in $\sigma\left(\tau_{w}\right)$.

Theorem 5.3. Let $V^{\prime}$ be the dual of a countably-Hilbert space $V$ which has a countable dense subset. Suppose $V^{\prime}$ be be endowed with a topology $\tau$. If $\tau$ is finer than $\tau_{w}$ and the inclusion map $i_{n}^{\prime}: V_{n}^{\prime} \rightarrow V^{\prime}$ is continuous for all $n$, then the $\sigma$-fields generated by $\tau$ and $\tau_{w}$ are equal (i.e. $\left.\sigma\left(\tau_{w}\right)=\sigma(\tau)\right)$. In particular, the $\sigma$-fields generated by the inductive, strong, and weak topologies on $V^{\prime}$ are equivalent (i.e. $\left.\sigma\left(\tau_{w}\right)=\sigma\left(\tau_{s}\right)=\sigma\left(\tau_{i}\right)\right)$. 
Proof. Let $U \in \tau$. Then $U_{n}=i_{n}^{\prime-1}(U)$ is open in $V_{n}^{\prime}$. By Proposition $5.1 U_{n}$ can be expressed as $U_{n}=\bigcup_{l \in T} x_{n_{l}}^{\prime}+F_{n}\left(\frac{1}{k_{l}}\right)$, where $x_{n_{l}}^{\prime} \in Q_{n}^{\prime}$ and $T$ is countable. Then

$$
U=U \cap V^{\prime}=U \cap\left(\bigcup_{n=1}^{\infty} V_{n}^{\prime}\right)=\bigcup_{n=1}^{\infty} U \cap V_{n}^{\prime}=\bigcup_{n=1}^{\infty} U_{n}=\bigcup_{n=1}^{\infty} \bigcup_{l \in T}\left(x_{n_{l}}^{\prime}+F_{n}\left(\frac{1}{k_{l}}\right)\right) \text {. }
$$

Thus $U$ can be expressed as a countable union of sets in $\sigma\left(\tau_{w}\right)$. Hence $U$ is in $\sigma\left(\tau_{w}\right)$. Therefore $\sigma\left(\tau_{w}\right)=\sigma(\tau)$.

Since $i_{n}^{\prime}$ is continuous with respect to $\tau_{i}$ and $\tau_{s}$ and also both $\tau_{i}$ and $\tau_{s}$ are finer than $\tau_{w}$, we see that the $\sigma$-fields generated by the inductive, strong, and weak topologies on $V^{\prime}$ are equivalent.

The $\sigma$-field on $V^{\prime}$ generated by the weak, strong, or inductive topology may be referred to as the Borel field on $V^{\prime}$.

\section{ACKNOWLEDGMENTS}

The author thanks professor Ambar Sengupta for his help and guidance in the research and revisions of this paper.

\section{REFERENCES}

1. Jeremy J. Becnel, About Countably-Normed Spaces, http://xxx.lanl.gov/abs/math.FA/0407200, 23 pages, 2004.

2. I.M. Gel'fand and G.E. Shilov, Spaces of fundamental and generalized functions, Generalized Functions, vol. 2, Academic Press, New York, New York, 1968. MR 0230128 (37:5693)

3. I.M. Gel'fand and N. Ya. Vilenkin, Application of harmonic analysis, Generalized Functions, vol. 4, Academic Press, New York, New York, 1964. MR0173945 (30:4152)

4. Gottfried Köthe, Topological vector spaces I, vol. 1, Springer-Verlag, Berlin, Germany, 1969. MR0248498 (40:1750)

5. Hui-Hsiung Kuo, White noise distribution theory, Probability and Stochastic Series, CRC Press, Inc., New York, New York, 1996. MR 1387829 (97m:60056)

6. A.P. Robertson and W.J. Robertson, Topological vector spaces, Cambridge University Press, London, 1964. MR0162118(28:5318)

7. Helmut H. Schaefer, Topological vector spaces, The Macmillan Company, New York, New York, 1966. MR0193469 (33:1689)

8. Yau-Chuen Wong, Introductory theory of topological vector spaces, Marcel Dekker, Inc., New York, New York, 1992. MR1198892 (94c:46003)

Department of Mathematics, Louisiana State University, Baton Rouge, Louisiana 70803

E-mail address: beck@math.lsu.edu 